\title{
DOUBLE EXCHANGE IN MIXED-VALENCY DILUTED MAGNETIC SEMICONDUCTORS
}

\section{J. BLINOWSKI}

Institute of Theoretical Physics, Warsaw University, Hoża 69, 00-681 Warszawa, Poland

\author{
AND P. KaCMaN
}

Institute of Physics, Polish Academy of Sciences

Al. Lotników 32/46, 02-668 Warszawa, Poland

The role of double-exchange, a non-Heisenberg spin-spin interaction between the magnetic ions of identical chemical nature but different charge states, in the mixed-valency diluted magnetic semiconductors is discussed. The mechanism correlates the spins of magnetic ions by virtual hopping of a $d$-electron from one ion to the other via the $p$-band states. We calculate the energy spectrum for pairs of magnetic ions within the charged ionic clusters stabilized by the charge-charge correlations, taking into account simultaneously both the double and the superexchange. The ferromagnetic, non-Heisenberg double exchange might compete with the Heisenberg-like, ferrimagnetic superexchange, thus resulting in canted alignments of ionic spins.

PACS numbers: 71.70.Gm, 75.30.Et

There are some members of the family of diluted magnetic semiconductors in which one can find coexisting magnetic ions of the same chemical nature but different charge state. The well known materials with this property are the zero-gap $\mathrm{A}^{\mathrm{II}} \mathrm{B}^{\mathrm{VI}}$ zinc-blende type semiconductors containing magnetic ions with the $2+/ 3+$ donor level degenerated with the conduction band. $\mathrm{HgSe}$ and $\mathrm{HgS}$ with Fe ions are the best studied examples of the above category (see [1] and the references therein), but possible candidates are also mercury chalcogenides with chromium and vanadium magnetic ions. Furthermore, coexisting different charge states of $\mathrm{Mn}$ ions were observed in $\mathrm{A}^{\mathrm{III}} \mathrm{B}^{\mathrm{V}}$ semiconductors $\left(\mathrm{Ga}_{1-x} \mathrm{Mn}_{x} \mathrm{As}\right.$, [2], and $\mathrm{In}_{1-x} \mathrm{Mn}_{x} \mathrm{As}$, [3]), even though the precise nature of the $3+\mathrm{Mn}$-related centers in these materials is not finally determined. Some authors suggest that these centers are complexes of $\mathrm{Mn}^{2+}$ ions with a tightly bound hole.

In diluted magnetic semiconductors (DMS) the dominant contribution to the ion-ion spin interactions comes from the superexchange mechanism mediated by the hybridization of ionic $d$-orbitals with the valence band states of the host crystal. The coexistence of the magnetic ions in different charge states opens a 
new channel of ion-ion exchange interactions resulting from the virtual hopping of the "extra" $d$-electron from one ion to the other. Such an interaction, termed the double exchange, was used by Anderson and Hasegawa to explain the magnetic properties of manganites of perovskite structure [4]. These authors considered a pair of $\mathrm{Mn}^{2+}-\mathrm{Mn}^{3+}$ ions with one $d$-electron hopping virtually from one ion to the other via the $p$-orbitals of the neighboring anions.

In this paper we consider a model $d^{4}-d^{5}$ pair of ions in a semiconductor crystal. In this case the internal exchange integral $J$ responsible for the intrashell correlations leading to the Hund rule is much larger than the anion-cation $s p-d$ hybridization integral, so that the perturbation theory can be used to derive the effective Hamiltonian for the low energy dynamics of the system. One has to account, however, for the fact that the virtual electronic states in a semiconductor are the band states rather than the localized anion levels. Using the $k$-space perturbation theory with respect to the $s p-d$ hybridization Hamiltonian we derive the effective Hamiltonian describing simultaneously the double exchange and the superexchange for a $d^{4}-d^{5}$ pair

$$
\widehat{H}_{\mathrm{eff}}=-2 J \widehat{S}_{1} \widehat{S}_{2}+\left[b\left(\widehat{a}_{2 l \sigma}^{+} \widehat{a}_{1 l \sigma}+\widehat{a}_{2 l(-\sigma)}^{+} \widehat{a}_{1 l(-\sigma)}\right)+\text { h.c. }\right] \text {. }
$$

$\widehat{S}_{1}$ and $\widehat{S}_{2}$ are the spin operators for $d$-electrons in the ion 1 and 2 , respectively. $\widehat{a}_{i l \sigma}^{+} \widehat{a}_{j l \sigma}$ are hopping operators transferring the electron with the spin $\sigma$ from the $l$-th $d$-orbital of the $j$-th ion to the $l$-th $d$-orbital of the $i$-th ion. The superexchange constant $J$ results from the fourth order perturbation theory. The double exchange constant $b$ comes already from the second order. Both constants depend, but in a different way, on the excitation energies of the system and involve summations over the valence bands in the entire Brillouin zone. They also differ in their dependencies on the interionic distance $\boldsymbol{R}_{1}-\boldsymbol{R}_{2}$. The corresponding formulae, as well as the evaluations of the numerical values of these constants will be reported elsewhere.

We start with the discussion of the case when the effective Hamiltonian (1) acts in the space of degenerate states, in which the unperturbed states $1 d^{4} 2 d^{5}$ and $1 d^{5} 2 d^{4}$ (i.e., the state with $4 d$-electrons on the ion 1 and 5 on the ion 2 , and vice versa) have the same energy. One can prove that the Hamiltonian (1) commutes with the operators of the square of the total spin of the pair $\widehat{S}^{2}=$ $\left(\widehat{S}_{1}+\widehat{S}_{2}\right)^{2}$ and of its $z$-component $\widehat{S}_{z}=\widehat{S}_{1 z}+\widehat{S}_{2 z}$, so that $S$ and $m_{S}$ remain good quantum numbers for the pair of the interacting ions. The diagonalization of the Hamiltonian (1) gives the energy spectrum of the pair in the form

where

$$
E_{ \pm}(S)=-J S(S+1) \pm \frac{|b|}{5} \sqrt{S(S+1)+1 / 4}+c
$$

$$
S=5 / 2+2=9 / 2,7 / 2, \ldots,|5 / 2-2|=1 / 2 .
$$

The states with lower energy $E_{-}(S)$, corresponding to the minus sign in formula (2), are, depending on the sign of the double exchange constant $b$, symmetric $(b<0)$ or antisymmetric $(b>0)$ combinations of the states $1 d^{4} 2 d^{5}$ and $1 d^{5} 2 d^{4}$. Regardless the sign of the constant $b$, the second order double exchange term favors the large values of the total spin of the pair. It has, therefore, essentially 
ferromagnetic though non-Heisenberg character. The superexchange term has a standard Heisenberg-like form, which, depending on the sign of the superexchange constant $J$, can be ferromagnetic $(J>0)$ or ferrimagnetic $(J<0)$. the ions.

For $J>0$ the lowest energy state of the pair is the state of parallel spins of

For $J<0$ there is a competition between the Heisenberg-like superexchange and non-Heisenberg double exchange terms. Minimizing the energy (2) with respect to the argument $S(S+1)+1 / 4$ we can distinguish three possible spin alignments corresponding to the lowest energy state, depending on the ratio $\mathcal{R}=b /(10 J)$

a) $|\mathcal{R}|>9 / 2$ the lowest energy is given by the maximum value of the total spin $S=9 / 2$ and, like in the $J>0$ case, the ferromagnetic alignment is privileged;

b) $|\mathcal{R}|<3 / 2-$ the lowest energy state corresponds to the minimum value of the total spin $S=1 / 2$ (ferrimagnetic, alignment);

c) $3 / 2<|\mathcal{R}|<9 / 2-$ the lowest energy state is a state with the intermediate values of the total spin $1 / 2<S<9 / 2$ (canted alignment),

The ratio $\mathcal{R}$ determines the magnetic properties of the pair, since the effect of the external magnetic field on the energy spectrum (2) reduces in the first approximation to an additive Zeeman-like term $g \mu_{\mathrm{B}} S_{z} B$ (we have to recall hene that this ratio is not unique in a given host material but may vary from pair to pair; owing to its dependence on the interionic distance). At low temperatures and strong magnetic fields one can expect steps in the pair contribution to the total magnetization in cases (b) and (c). It is interesting to observe, however, that the double exchange has no effect on the pair contribution to the high temperature susceptibility of the system, which is completely determined by the superexchange, even in the ferromagnetic case (a), i ie, for large values of $b$. This is due to the cancellation of the terms with plus and minus sign in Eq. (2) in the high temperature expansion.

We pass now to the case in which the local potential energy fluctuations: remove the degeneracy between the states $1 d^{4} 2 d^{5}$ and $1 d^{5} 2 d^{4}$. We denote the energies of these states by $E_{1}$ and $E_{2}>E_{1}$, respectively. In this case the energy spectrum of the pair has a more complex form

$E_{ \pm}(S)=-J S(S+1)+\frac{E_{1}+E_{2}}{2} \pm \sqrt{\left(\frac{E_{2}-E_{1}}{2}\right)^{2}+\frac{b^{2}}{25}[S(S+1)+1 / 4]+c}$

In the limiting case when the energy splitting $E_{2}-E_{1}$ is much larger than the double exchange term the square root may be expanded and we get the purely Heisenberg form of the spin-spin interaction

$$
E_{ \pm}(S)=-J S(S+1)+\frac{E_{1}+E_{2}}{2} \pm \frac{E_{2}-E_{1}}{2} \pm \frac{b^{2}[S(S+1)+1 / 4]}{25\left(E_{2}-E_{1}\right)}
$$

which gives the lowest energy state in the form

$$
E_{-}=E_{1}-\left[J+\frac{b^{2}}{25\left(E_{2}-E_{1}\right)}\right] S(S+1)
$$

In this limit the double exchange is still ferromagnetic, but strongly suppressed as 
compared to the degenerate case, and represents only a minor correction to the dominating superexchange integral.

The description of the macroscopic magnetic properties of semiconductors with coexisting magnetic ions in different charge states represents a difficult theoretical problem. The spatial distribution of the ions carrying the extra charge is not random. It depends very much on the character of the compensating charge. In this paper we have presented the detailed discussion of the spin-spin interactions within a $d^{4}-d^{5}$ pair of magnetic ions in a semiconductor. Such a pair model represents only a first step towards understanding the magnetic properties of mixed valency DMS's, shining light on the microscopic mechanisms of spin-spin interactions in these materials. We believe, however, that there are some specific situations, in which the pair model might be of more direct use:

1. In zero-gap materials the extra charge of magnetic donors is compensated by the free carriers in the conduction band, quasiuniformly smeared out over the entire crystal. As shown by Wilamowski et al. [5], the charge-charge correlations keep the charge donors as far as possible from each other. The position of each extra charge is not completely fixed, however. When two (or more) ions are situated in the vicinity of the local potential energy minimum, the extra charge is bound by a pair (or larger cluster) rather than by an individual ion. One may expect the properties of such small clusters to be properly described by the discussed in this paper pair model or by its simple generalization for slightly larger clusters.

2. In $\mathrm{A}^{\mathrm{III}} \mathrm{B}^{\mathrm{V}}$ materials with $\mathrm{Mn}$ ions the $\mathrm{Mn}^{2+}$ centers are negatively charged compared to the other cations. In $n$-type materials this extra charge is compensated by ionized donors. In this situation negatively charged are those manganese ions which are nearest to the donors. Here again the pair model might be useful for the description of the magnetic properties of pairs formed by the $\mathrm{Mn}^{2+}$ ions with the nearby $\mathrm{Mn}^{3+}$ centers. The conclusions of the $d^{4}-d^{5}$ pair model should remain qualitatively correct, in our opinion, even in the case when the $\mathrm{Mn}^{3+}$ centers represent complexes " $\mathrm{Mn}^{2+}+$ hole", provided the spin of the hole is strongly coupled to the spin of the ion.

\section{Acknowledgments}

This work was partially supported by the Committee for Scientific Research, grant No. 2 P03B 02511.

\section{References}

[1] Z. Wilamowski, Acta Phys. Pol. A 77, 133 (1990).

[2] X.C. Liu, D. Heiman, J. Hao, K.C. Hsieh, Mater. Sci. Forum 182-184, 627 (1995).

[3] H. Munekata, H. Ohno, S. von Molnar, A. Segmüller, L.L. Chang, L. Esaki, Phys. Rev. Lett. 63, 1849 (1989).

[4] P.W. Anderson, H. Hasegawa, Phys. Rev. 100, 675 (1955).

[5] Z. Wilamowski, K. Świątek, T. Dietl, J. Kossut, Solid State Commun. 74, 833 (1990). 